livraisons

d'Histoire

de l'Architecture

\section{Livraisons de l'histoire de l'architecture}

$31 \mid 2016$

La source photographique dans la pratique de I'historien de l'architecture

\title{
Les fonds photographiques des Archives nationales, une source pour l'historien de l'architecture
}

Photographical funds of the French Archives nationales, a source for history of architecture

Die fotografischen Bestände der Archives Nationales, eine Quelle für die Architekturgeschichte

\section{Sandrine Bula}

\section{(2) OpenEdition}

Journals

Édition électronique

URL : http://journals.openedition.org//ha/597

DOI : 10.4000//ha.597

ISSN : 1960-5994

\section{Éditeur}

Association Livraisons d'histoire de l'architecture - LHA

Édition imprimée

Date de publication : 14 juillet 2016

Pagination : 81-90

ISSN : 1627-4970

Référence électronique

Sandrine Bula, "Les fonds photographiques des Archives nationales, une source pour l'historien de l'architecture ", Livraisons de l'histoire de l'architecture [En ligne], 31 | 2016, mis en ligne le 14 juillet 2018, consulté le 20 avril 2019. URL : http://journals.openedition.org//ha/597 ; DOI : 10.4000//ha.597

Ce document a été généré automatiquement le 20 avril 2019.

Tous droits réservés à l'Association LHA 


\section{Les fonds photographiques des Archives nationales, une source pour l'historien de l'architecture}

Photographical funds of the French Archives nationales, a source for history of architecture

Die fotografischen Bestände der Archives Nationales, eine Quelle für die Architekturgeschichte

\section{Sandrine Bula}

1 La photographie entretient avec l'architecture d'étroites relations: le monument constitua l'objet idéal d'une technique exigeant à ses débuts forte luminosité et long temps de pose ${ }^{1}$. Elle est devenue très tôt l'auxiliaire de l'architecte ${ }^{2}$, utilisée pour documenter un chantier de construction ou de restauration, reproduire des plans (« bleus », tirages sur papier albuminé...), tandis que la pratique du photomontage permet de rendre visible et de contextualiser un projet. Les photographies sont exécutées par l'architecte, des praticiens ou studios spécialisés, des entreprises trouvant là un moyen de promouvoir leur savoir-faire. Elles accompagnent un rapport, illustrent un article destiné aux professionnels ou au grand public.

On trouvera aux Archives nationales des photographies correspondant à ces typologies et usages, dans les fonds des services chargés de l'entretien du patrimoine immobilier de l'État ou de la mise en œuvre de sa politique en matière d'architecture et d'urbanisme, comme dans ceux d'architectes ayant confié leurs archives à l'institution.

Mais les sources photographiques ne se cantonnent pas aux seuls fonds directement liés à la pratique architecturale, elles sont aussi présentes, parfois de façon incidente, dans de nombreux autres fonds d'origine publique ou privée.

4 S'il existe des ensembles iconographiques homogènes et volumineux pouvant être considérés comme des fonds photographiques à part entière, l'on se trouve souvent face à des séries de dossiers incluant des photographies en nombre variable, comme à de 
nombreuses pièces isolées ou en petits groupes, disséminées dans de multiples séries et versements.

5 L'objet de la présente contribution ne saurait être un recensement exhaustif mais plutôt la mise en perspective d'ensembles présentant une certaine cohérence et continuité, ainsi que le signalement de pistes sur lesquelles le chercheur pourrait s'engager.

\section{Les bâtiments civils et palais nationaux}

6 L'histoire de l'administration en charge du patrimoine immobilier de l'État est assez complexe, au gré des éclatements ou fusions de services. On peut cependant y distinguer une continuité, incarnée par le service des Bâtiments civils et Palais nationaux, créé sous la Révolution, en charge de la construction des édifices publics et de l'entretien des anciens palais royaux. Il constituera une structure stable de l'administration des BeauxArts, jusqu'à la création du ministère des Affaires culturelles en 1959. Au sein de ce dernier, il dépendra, jusqu'à sa suppression à la fin des années 1970, d'une direction de l'Architecture.

7 Quant au service des Monuments historiques, apparu en 1830 avec la nomination d'un premier inspecteur, Ludovic Vitet, il assurera lui-même la conservation de ses archives, considérées comme toujours vivantes, les Archives nationales n'étant destinataires que de dossiers de carrière du personnel.

8 Le service des Bâtiments civils et Palais nationaux comprend deux entités. La première, le conseil des bâtiments civils, devenu « conseil des bâtiments de France » en 1945 jusqu'à sa disparition en 1970, examine les projets de travaux. Ses archives ne comportent pas de photographies. La seconde, le service des Bâtiments civils proprement dit, verra son périmètre d'action se rétrécir au cours du temps, les ministères et collectivités locales décidant des travaux pour les bâtiments dont ils ont la propriété ou l'usage : casernes, palais de justice, préfectures, asiles d'aliénés, hôtels de ville et autres établissements communaux hôpitaux et hospices départementaux, édifices diocésains, prisons, facultés et lycées, hôtels des monnaies ou des postes etc.

9 La liste des bâtiments civils et des palais nationaux variera et s'étoffera au cours du temps. Y figureront également l'église abbatiale de Saint-Denis, la Sainte-Chapelle, la cathédrale Notre-Dame de Paris, le Panthéon.

10 Les archives du service des Bâtiments civils et Palais nationaux se trouvent dans la soussérie $\mathrm{F} / 21$, créée en 1906 pour accueillir les premiers versements de l'administration des Beaux-Arts, ainsi que dans la série VA (versement de l'Architecture), entrée aux Archives nationales en 1960, et qui se présente sous la forme de 216 cotes de recueils de plans et documents iconographiques classés par édifices.

11 Peu de photographies sont conservées dans les dossiers de $\mathrm{F} / 21^{3}$, en raison de leur nature même : correspondance avec les architectes en charge des édifices, rapports aux autorités de tutelle, mémoires d'ouvrages de maçonnerie et cahiers d'attachements produits par les architectes. Il s'agit de contrôler les opérations et la bonne utilisation des crédits. Ainsi, les dossiers concernant le Louvre et les Tuileries ${ }^{4}$ évoquent les travaux d'entretien, l'utilisation des locaux par divers services dont le ministère des Finances, l'organisation de fêtes et cérémonies, le personnel, l'éclairage, les attractions et jeux dans les jardins, sans qu'aucune photographie ne soit jointe au dossier, de même que les dossiers consacrés exclusivement aux travaux de la réunion des deux palais de 1852 à $1865^{5}$. Une 
exception pour Versailles: deux tirages coloriés représentant le bassin de Neptune, provenant d'un entrepreneur d'illuminations, relatif à des festivités nocturnes organisées en juillet 1904 par la Société des fêtes versaillaises ${ }^{6}$.

12 Il faut plutôt chercher les photographies dans la série VA, qui en comporte 136, relatives à 28 édifices. La plus ancienne, datée de 1855 et signée d'Henri Le Secq, représente une façade du château de Saint-Germain-en-Laye ${ }^{7}$, les plus récentes, de 1946, concernent un projet de centre scolaire d'éducation physique à Arcachon.

13 Ce fonds ne comporte aucune photographie du Louvre, mais dix se rapportent aux Tuileries avant leur incendie (il s'agit cependant de reproductions photographiques de dessins) ${ }^{8}$, ainsi qu'à des projets de bâtiments d'attractions et à l'installation provisoire dans les jardins de la Bibliothèque Schoelcher, dans les années 1886-1888.

14 Les plans prédominent dans la série VA : ainsi, le recueil du Panthéon ne comporte qu'une seule photographie sur 141 pièces ${ }^{9}$. Il est difficile de savoir qui a collecté des documents, à quel moment, et pour quel usage précis: on note par exemple que Louis Clémentin Bruyerre, inspecteur aux grands travaux des Tuileries de 1861 à $1865^{10}$, a rassemblé des documents relatifs à ce palais ${ }^{11}$.

15 Face à l'accroissement de la masse des affaires à suivre vont se constituer des services déconcentrés: les conservations régionales des bâtiments de France dans les années 1950-60 et les agences départementales des bâtiments de France, en 1946.

16 Au ministère de la Culture, la sous-direction des Bâtiments civils se maintient sous diverses appellations. Elle perd en 1968 la gestion des palais nationaux au profit de la sous-direction des Monuments historiques. Des versements de cette dernière ont été effectués aux Archives nationales. Les dossiers d'édifices comportent souvent des photographies à l'appui des rapports des architectes ${ }^{12}$.

17 La sous-direction des Affaires générales et des constructions publiques (dénomination apparue en 1979) garde les bâtiments civils et ne disparaît qu'en 1990, lors de la création du service national des travaux (SNT).

On peut observer dans les dossiers de la sous-direction un emploi courant et systématique de la photographie à l'appui des rapports et mémoires fournis par les architectes avec, dans les années 1950-1960, une certaine uniformisation de la présentation. Tel est le cas du dossier des travaux entrepris à l'hôtel Montalivet situé rue de Varenne à Paris, se rapportant à l'entretien et aux grosses réparations de 1954 à 1977 (ill. 1). Il comporte trois parties distinctes: photos, plans, textes. Huit photographies documentent l'état du bâtiment et les travaux entrepris en 1948-1949. 


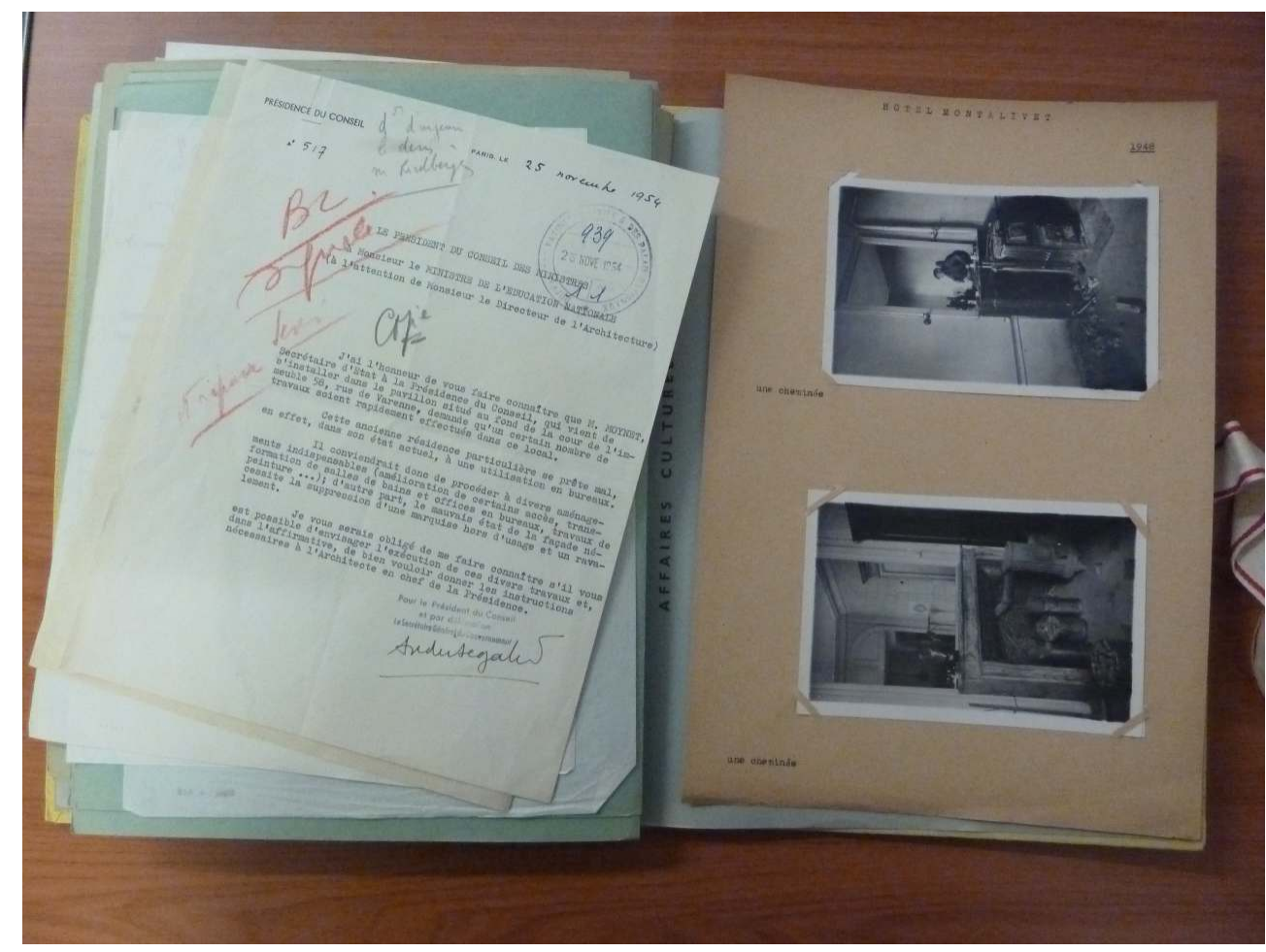

Tirages gélatino-argentiques 9,5 × 14,5 cm montés sur carton 32 × 22 cm, Archives nationales, 19880557/123.

(c) Cl. S. Bula émaner de sources diverses témoignant d'un état antérieur du bâtiment (archives photographiques de la Caisse nationale des monuments historiques et des sites, pour l'hôtel Montalivet ${ }^{13}$, cartes postales anciennes pour la cour d'appel de Bastia ${ }^{14}$, Service américain d'information pour le Quai d'Orsay au moment de la Libération ${ }^{15}$ ).

Au Service national des travaux s'ajoute en 1998 l'Établissement public de maîtrise d'ouvrage des travaux culturels (EMOC): les deux organismes fusionnent dans un établissement public à caractère administratif chargé de la construction publique, l'Opérateur du patrimoine et des projets immobiliers de la Culture, ou OPPIC, créé en 2010.

On trouve dans ces archives récentes des dossiers élaborés par les architectes menant les travaux : ils peuvent être riches en photographies documentant toutes les zones et étapes d'un chantier. Ainsi, la restauration de l'amphithéâtre de Verniquet du Muséum d'histoire naturelle à Paris comporte plus de 200 tirages numériques ${ }^{16}$. Les pratiques évoluent: il ne s'agit plus de joindre des tirages à l'appui du texte, l'usage de la photographie numérique permet désormais d'incorporer aux rapports un grand nombre d'illustrations. 


\section{Les agences publiques d'architecture}

\section{d'architecture du Grand Palais 17 comportent des plans, des dessins et de nombreuses} photographies, souvent des grands formats fragiles, dont les cartons de montage sont endommagés. L'historien de l'architecture y trouvera des témoignages intéressants sur la construction de l'édifice, en particulier sur la prouesse technique que fut l'installation de la charpente métallique et la verrière de la grande nef, et la structure métallique de la nef du Palais de l'Industrie, construit à l'occasion de l'Exposition universelle de 1855, puis démoli pour laisser la place aux Grand Palais et Petit Palais, en vue de l'Exposition universelle de 1900. Le fonds conserve également des photographies relatives à la Galerie des Machines construite face à l'École militaire dans le cadre de l'Exposition universelle de 1889.

Le fonds de l'agence d'architecture du Louvre et des Tuileries (64 AJ), résulte du regroupement en 1854 de l'agence des grands travaux du Louvre, créée en 1848, et de l'agence de réunion des Tuileries au Louvre créée par l'architecte Visconti en 1852 (ill. 2). Ce fonds comporte huit albums contenant 1100 photographies se rapportant à l'ensemble et détails des façades du nouveau Louvre, pour les années 1852-1857 ${ }^{18}$. Les œuvres des sculpteurs ornemanistes et statuaires ayant travaillé au Louvre sous le Second Empire sont aussi représentées par près de 3600 pièces $^{19}$. À cela s'ajoute un ensemble d'environ 500 photographies, depuis 1854 environ jusqu'aux années $1960^{20}$, formant un riche corpus documentaire. On y notera la présence de plusieurs photographies d'Édouard Baldus chargé par l'architecte Lefuel de suivre l'évolution du chantier ${ }^{21}$. 


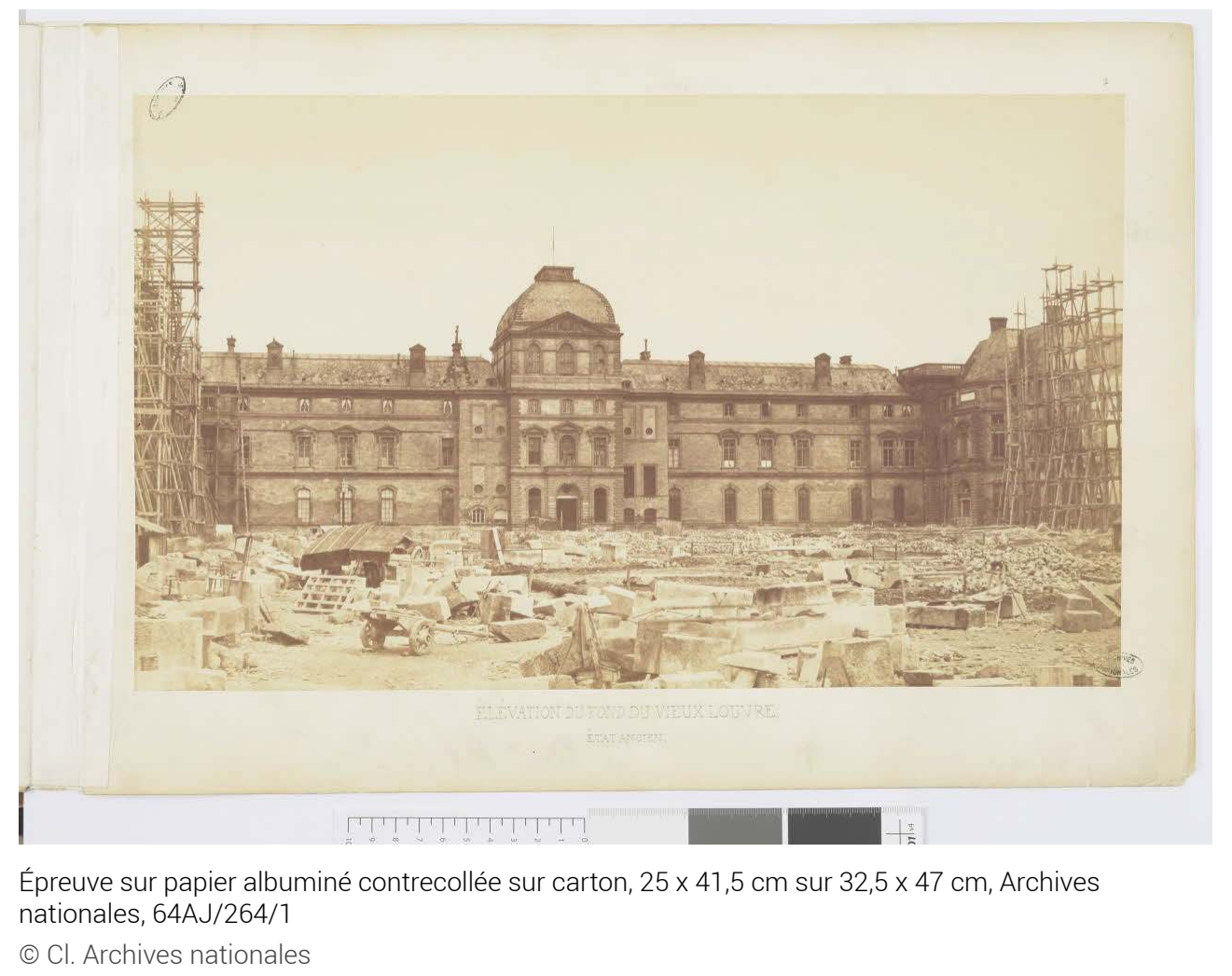

Par la suite, les architectes en chef des bâtiments civils et palais nationaux chargés du domaine du Louvre et des Tuileries verseront aux Archives nationales leurs dossiers, dans lesquels on retrouve également des photographies ${ }^{22}$.

\section{Les édifices cultuels}

À côté des séries dévolues aux bâtiments civils, une autre source photographique est identifiable: celle des dossiers d'édifices cultuels. Dans le système concordataire, la gestion des cultes revint, à partir de 1839, à une direction des Cultes autonome, rattachée tantôt au ministère de la Justice, de l'Intérieur ou de l'Instruction publique, compétente pour l'entretien des édifices religieux appartenant à l'État.

Pour gérer les questions de bâtiments, on créa en 1848 le corps des architectes diocésains, chargés des travaux nécessaires aux cathédrales, évêchés et séminaires et du contrôle des travaux sur les édifices paroissiaux ou consistoriaux que la direction des cultes subventionnait. En 1853 fut institué un comité des Inspecteurs généraux des édifices diocésains, chargés de visiter, inspecter et d'examiner les plans et devis fournis par les architectes diocésains. Les archives issues du comité s'interrompent en 1879.

Pour la période 1853-1879, les Archives nationales conservent les avis et rapports se rattachant aux procès verbaux des séances tenues par le comité des inspecteurs généraux et les pièces jointes ${ }^{23}$, qui ne comportent pas de photographies, et les dossiers relatifs aux travaux exécutés aux séminaires diocésains, évêchés et cathédrales, qui eux en conservent, jointes aux rapports ${ }^{24}$. Les photographies d'édifices sont présentes dans les dossiers, conformément à ce que préconisait le ministère de l'Instruction publique et des 
Cultes dans une instruction du 24 juillet 1848 «pour la rédaction des projets, l'exécution des travaux de la rédaction des mémoires concernant les édifices religieux » : " Il serait même à propos de produire autant que possible des vues prises au daguerréotype des principaux aspects des bâtiments à réparer ${ }^{25}$. » Un exemple de ces daguerréotypes nous est parvenu, dans le dossier de la cathédrale de Saint-Brieuc ${ }^{26}$.

Après la séparation de l'Église et de l'État en 1905, les cultes luthérien, réformé et israélite recueillent leurs anciens biens et en deviennent propriétaires. La charge des églises est confiée aux communes, le service des Édifices diocésains intègre le service des Monuments historiques en 1907. La direction des Cultes disparaît et l'intervention de l'État se réduit à l'activité d'un simple bureau du ministère de l'Intérieur qui, à partir de 1911, gère les questions relatives aux édifices cultuels (statut, secours et subventions pour des réparations). Les dossiers comportent le plus souvent quelques photographies de l'édifice, des dégradations constatées, à l'appui des demandes de subvention ${ }^{27}$.

\section{Les archives d'architectes : fonds d'origine privée}

Les Archives nationales conservent également une vingtaine de fonds d'architectes, dont une quinzaine comportent des photographies. De nombreux fonds de cette nature ont été orientés vers l'Institut français d'architecture (IFA) ${ }^{28}$. D'autres fonds d'agences d'architectes sont conservés aux Archives nationales du monde du travail (ANMT) à Roubaix : ceux de Stanislas Fiszer, ou encore de Dominique Perrault.

Ces archives recèlent logiquement des photographies documentant les projets et réalisations, mais également des photographies purement personnelles et familiales ou encore d'autres à la frontière entre vie privée et activité professionnelle, qui témoignent des goûts et centres d'intérêt, ou encore de liens de travail et d'amitié de l'architecte.

De ce point de vue, les fonds de Paul et Marion Tournon présentent un intérêt particulier ${ }^{29}$, en raison du nombre des photographies conservées comme de leur variété : projets et chantiers, documentation, villégiatures et de voyages d'étude, supports pédagogiques. De même, le fonds d'Albert Laprade comporte plus de 2500 photographies concernant pour la plupart ses réalisations ${ }^{30}$.

L'on peut citer, par ordre décroissant du nombre de photographies conservées : les fonds Walter (357 AP : 630 photographies), Sanson (143 AP : 620 photographies), Baltard (332 AP : 353 photographies, dont une trentaine sous la forme de portraits «carte de visite » de relations et d'amis, et 152 vues de Venise et Rome). Le fonds Labrouste (453 AP) ne compte, lui, que 15 photographies, qui ne représentent pas des œuvres d'Henri Labrouste, mais notamment des villas édifiées par son fils Léon, dans les années 1880-1890.

\section{Urbanisme et Reconstruction, Tourisme}

Avec la photothèque du ministère de l'Urbanisme et de la Reconstruction (1945-1949) ${ }^{31}$, il ne s'agit plus de pièces incluses dans les dossiers de bâtiments particuliers, mais d'un ensemble volumineux destiné à rendre compte de l'action de l'État, à l'usage des services du ministère en tant que source de documentation et outil de communication. 
Un autre ensemble intéressant un aspect particulier de l'histoire de l'architecture est constitué par les photographies relatives aux Expositions universelles, conservées dans la sous-série F/12 (ministère du Commerce et de l'Industrie). Les archives émanant du commissariat général et des services du ministère en charge de l'organisation des Expositions de 1855, 1878, 1889 et 1900 ont été versées entre 1877 et 1931, au terme de sévères éliminations, y compris de photographies, notamment pour l'exposition de $1900^{33}$. Un corpus iconographique unissant plans, dessins et photographies des pavillons a été constitué. Il n'inclut pas de photographies relatives à l'exposition de 1855. Il s'agit souvent de tirages largement diffusés à l'époque, provenant de studios photographiques connus : Braun, Pierre Petit, Eugène Pirou, etc. ${ }^{34}$. Pour l'Exposition universelle de $1937^{35}$, l'on dispose d'une série d'albums documentant les différentes phases du chantier. La participation de la France à des expositions récentes, telle celle de Séville en 1992 est aussi représentée par un certain nombre de photographies, dans les dossiers produits par la compagnie française pour l'Exposition de Séville ${ }^{36}$.

42 Pour les $\mathrm{XX}^{\mathrm{e}}$ et $\mathrm{XXI}^{\mathrm{e}}$ siècles, les sources deviennent foisonnantes : des photographies se rapportant aussi bien aux établissements pénitentiaires, d'enseignement, qu'à la question des bidonvilles en France ou en Algérie peuvent être mises à profit par l'historien de l'architecture ${ }^{37}$.

43 De nouveaux fonds riches de photographies parviennent chaque année aux Archives nationales (le musée d'Orsay a versé en 2016 le fonds de son secrétariat général, comportant plus de 700 photographies relatives à la transformation de la gare d'Orsay en musée). Grâce à un travail continu et approfondi de signalisation, de description des documents et de mise en ligne progressive d'instruments de recherche, il est possible, aujourd'hui, d'accéder plus facilement à la multitude de photographies que recèlent les 
fonds des Archives nationales. C'est ainsi que la reprise sous format électronique de l'inventaire d'une sous-série dévolue aux papiers d'érudits et pièces isolées a permis de repérer 59 photographies des anciennes prisons de Paris, passées longtemps inaperçues ${ }^{38}$ : l'historien de l'architecture a encore devant lui de belles découvertes et redécouvertes.

\section{NOTES}

1. Giovanni Fanelli, Storia della fotografia di architettura, Roma, Laterza, 2009, $457 \mathrm{p}$.

2. Manuel de traitement des archives d'architecture, XIXe-XXe siècles, Conseil international des archives, Paris, 2000, $144 \mathrm{p}$.

3. Les cotes concernant les édifices à partir de la seconde moitié du XIXe siècle sont: F/21/2990-2469, (2873)-2907-3500/11, 3701-3966/4, 7123-7220. On trouvera l'historique des différents services dans les introductions des instruments de recherche de la série F/21 en ligne dans la salle des inventaires virtuelle des Archives nationales.

4. $\mathrm{F} / 21 / 2334-2338,7147-7152$.

5. $\mathrm{F} / 21 / 1691-1759$.

6. $\mathrm{F} / 21 / 2422$.

7. Le Secq a photographié le château en 1850 et en 1855. Voir Eugenia Parry et Josiane Sartre, Henri Le Secq, photographe de 1850 à 1860 : catalogue raisonné de la collection de la Bibliothèque des arts décoratifs, Paris, Flammarion, 1986, $191 \mathrm{p}$.

8. $\mathrm{CP} / \mathrm{VA} / 15$, p. 38-41, 79-83.

9. $\mathrm{CP} / \mathrm{VA} / 16$, p. 139, photographie de Marville.

10. Voir son dossier de Légion d'honneur : LH/386/41.

11. Voir l'inventaire analytique dactylographié de la VA/1 à VA/216 par Catherine Grodecki et Georges Bailhache, 1962, 361 p., p. 15.

12. Par exemple, dans le versement $19900057 / 27$ : dossier du château de Pau, 1961-1984.

13. $19880557 / 123$.

14. $19880557 / 73$.

15. $19880557 / 124$.

16. $20130464 / 81$. Archives produites par l'EMOC et versées par l'OPPIC. Dossier documentaire, 2001-2004.

17. $102 \mathrm{AJ}$. Sa cotation est en cours de révision.

18. $64 \mathrm{AJ} / 263 / 1$ à $64 \mathrm{AJ} / 266 / 2$.

19. $64 \mathrm{AJ} / 271$ à $285 /$ bis. Ces articles conservent aussi exclusivement des photographies, à l'exception des articles 280 à 282 composés également de dessins et de correspondances.

20. $64 \mathrm{AJ} / 286 / 1$ à 286/43.

21. Voir Le Photographe et l'architecte: Édouard Baldus, Hector-Martin Lefuel et le chantier du nouveau Louvre de Napoléon III, Paris, Réunion des musées nationaux, 1995, 135 p.

22. On trouve les archives des architectes Marc Saltet, Jean-Claude Daufresne, Georges Duval et Guy Nicot dans les versements 19960289 ; 20020209 et 20020432, pour les années 1968-2001.

23. F/19/4546 à 4556 (édifices diocésains et paroissiaux). On trouvera l'historique des différents services dans les introductions des instruments de recherche de la série F/19 en ligne dans la salle des inventaires virtuelle des Archives nationales.

24. $\mathrm{F} / 19 / 7286$ à $\mathrm{F} / 19 / 7926$. 
25. Cité dans Malcolm Daniel et Barry Bergdoll, The Photographs of Édouard Baldus, The Metropolitan museum of art, 1994, 293 p., p. 105, 275. Voir aussi Jean-Michel Leniaud, Béatrice Bouvier, Le Livre d'architecture, XVe-XXe siècles, Paris, École nationale des chartes, 2002, 335 p., p. 275.

26. $\mathrm{F} / 19 / 7864$.

27. Pour les subventions allouées aux communes pour l'entretien des édifices cultuels leurs appartenant, voir F/19/20001-20211 (1941-1968) ; 19770131/1-28 (1964-1970).

28. Association créée en 1981 pour assurer la promotion de l'architecture contemporaine française. Elle est aujourd'hui l'un des trois départements de la Cité de l'architecture et du patrimoine, devenue établissement public. Elle s'est vu confier, en 1986, par la direction des Archives de France, la mission de «centre intermédiaire de traitement des archives d'architecture du XXe siècle auprès des Archives nationales ». Pour assurer cette mission, un Centre d'archives d'architecture du XXe siècle a été créé rue de Tolbiac à Paris. Ouvert au public en 1989 , il conserve à ce jour près de 400 fonds.

29. Le fonds de Paul Tournon est coté 377 AP et conserve plus de 5000 photographies; les archives de sa fille, Marion Tournon-Branly, sont constituées de 13 versements dont 6 constitués de phototypes (20060041: négatifs noir et blanc; 20060042: photographies noir et blanc; 20060043 : diapositives couleur; 20060044 : diapositives noir et blanc en verre; 20060045 : négatifs couleur ; 20060224 : photographies couleur).

30. $403 \mathrm{AP}$.

31. Voir les fiches de recherche $n^{\circ} 63:$ "La photothèque du ministère de l'Urbanisme et de la Reconstruction, 1944-1971 » et $n^{\circ} 102$ : « la photothèque du Tourisme », disponibles en salle des inventaires virtuelle des Archives nationales (onglet « conseils pour la recherche »).

32. Sous les cotes $\mathrm{F} / 14 / 18261$ à $\mathrm{F} / 14 / 18365$.

33. Voir l'introduction de: Expositions universelles internationales et nationales au XIXe siècle, répertoire numérique détaillé sélectif des articles de la sous-série F/12 relatifs aux expositions, An VI-1914, par Marc Smith, Paris, Archives nationales, 1994, 159 p. (Cotes décrites : F/12/985 à 991, 2892 à 4475,4977 à 5068, 5793 à 5813, 6635 à 6670, 7510 à 7587, 8810 à 8847, 11849 à 11927).

34. Voir aussi : Exposition universelle de 1867 à Paris: documents iconographiques. Inventaire, par Christiane Douyère-Demeulenaere, Paris, Archives nationales, 2003, 26 p. (Cotes décrites: $\mathrm{F} / 12 / 11869$ à 11893).

35. Exposition internationale de 1937 à Paris. Répertoire numérique détaillé, dact. par A. Labat, 1994, 177 p. (Photographies : F/12/12114 à 12118).

36. Versements 19930322, 19930662, 19940299, 19950020. Cette structure dépendait du ministère de l'Économie et des Finances.

37. Voir la fiche de recherche $n^{\circ} 30$ : "Sources photographiques: les fonds du XXe siècle", disponible en salle des inventaires virtuelle des Archives nationales (onglet « conseils pour la recherche »).

38. $\mathrm{AB} / \mathrm{XIX} / 333$.

\section{RÉSUMÉS}

Les ressources photographiques que les Archives nationales peuvent offrir dans le domaine de l'architecture sont réparties dans les fonds constitués par les administrations centrales et les 
organismes en charge des édifices publics. Les dossiers produits par le service des bâtiments civils et palais nationaux comportent souvent des photographies, dont les plus anciennes remontent à la seconde moitié du XIX ${ }^{\mathrm{e}}$ siècle. Un ensemble particulièrement important est formé par les photographies de l'agence d'architecture du Louvre et des Tuileries, qui documentent les travaux entrepris sous le Second Empire. Les archives des services des ministères en charge des cultes conservent, eux aussi, un ensemble significatif de photographies. Des fonds d'architectes d'origine privée (fonds Laprade, Sanson, Tournon...) représentent une source non négligeable, de même que de nombreux autres fonds (ministère de la Reconstruction, du Tourisme, dossiers du ministère de l'Industrie relatifs aux Expositions universelles...) proposent aux chercheurs un abondant matériau iconographique souvent inédit.

The national Archives's photographical ressources are dispatched into the funds created by central administrations and organisations in charge of public structures. The files produced by the civil buildings's service and national palaces often contain photographs, amongst which the oldest are dated back to the second half of the nineteenth century. A particularly important part is based on the photographs by the Louvre and the Tuileries' architecture agency which documented the works done under the Second Empire. The archives of the ministry of Interior in charge of the religious architecture also preserve a significant amount of photographs. Former private architects's funds (Laprade, Sanson, Tournou...) represent a substantial source, alongside many other funds (ministry of Reconstruction, Tourism, files related to Universal Expositions from the ministry of Industry...) offer a rather exclusive and abundant iconographic material for scholars.

Die architekturgeschichtlich relevanten Bestände der Archives Nationales setzen sich zusammen aus Quellen der zentralen Verwaltungsbehörden sowie Beständen der für die öffentlichen Bauten zuständige Stellen. Darunter beinhalten insbesondere die Akten des Service des Bâtiments Ciils et palais nationaux zahlreiche Fotografien, die teilweise bis ins 19. Jahrhundert zurückreichen. Ein besonders bedeutendes Ensemble stellen die Aufnahmen der Agence d'architeture du Louvre et des Tuileries dar, in denen die während des Second Empire durchgeführten Arbeiten dokumentiert werden. Auch enthalten die Bestände der für Kultusangelegenheiten zuständigen Abteilungen des Innenministeriums umfangreiche Fotosammlungen. Darüber hinaus bestehen bedeutende Bestände in Privatsammlungen (Laprade, Sanson, Tournon usw.), die eine nicht zu vernachlässigende Quelle darstellen, sowie zahlreiche weitere Fotosammlungen (Ministère de la Reconstruction, Ministère du Tourisme, Akten des Industrieministeriums mit Bezug auf die Weltausstellungen usw.), die für die Forschung ein umfangreiches, großenteils noch unerforschtes Material bieten.

\section{AUTEUR}

\section{SANDRINE BULA}

Sandrine Bula est conservateur du patrimoine, responsable de la Mission photographie des Archives nationales. Adresse électronique : sandrine.bula@culture.gouv.fr 